\title{
Discrete Event Simulation of QoS of a SCADA System Interconnecting a Power Grid and a Telco Network
}

\author{
Ester Ciancamerla ${ }^{1}$, Chiara Foglietta ${ }^{1}$, Davide Lefevre ${ }^{1}$, \\ Michele Minichino ${ }^{1}$, Leonid $\mathrm{Lev}^{2}$, and Yosi Shneck ${ }^{2}$ \\ ${ }^{1}$ ENEA, via Anguillarese 301, 00100 Rome, Italy \\ ${ }^{2}$ Israel Electric Corporation, Haifa, Israel \\ ester.ciancamerla@enea.it, chiara.foglietta@enea.it, \\ davide.lefevre@enea.it, michele.minichino@enea.it, \\ leonid.lev@iec.co.il, shneck.yosi@gmail.com
}

\begin{abstract}
Indicators of Quality of Service (QoS) of Fault Isolation and System Restoration (FISR) service, delivered by SCADA system are computed, discussed and correlated to quality indicators of power supplied to customers. In delivering FISR service, SCADA system, Telco network and Power grid act as a whole heterogeneous network. While SCADA system and Telco network can be well represented by means of discrete event simulators. To represent a Power grid a continuous simulator is typically required. In the paper, to compute QoS of FISR, SCADA system, Telco network and Power grid have been represented by a unique model by means of a discrete event simulator.
\end{abstract}

Keywords: Critical Infrastructures, SCADA, Quality of Service, Discrete Event Simulation.

\section{Introduction}

SCADA systems constitute the nervous systems of Power grids. They rely on SCADA communication links which are dependent upon Telco networks and represent one of the major channels for mutual propagation of disturbances and adverse events between Power grids and Telco networks. Power grids and Telco networks have a heavy impact on daily life and are typically referred to as Critical Infrastructures (CIs), since their correct operation is essential for the everyday life of our modern society. Dependent (bi)directional relationships and reciprocal influences among CIs are named (inter)dependencies. CIs are more and more reliant on information and communication technology and, largely through this reliance they have become more and more interdependent [1].

There is a growing interest in developing models and tools for CI interdependency analysis. In literature, a huge extension of modeling approaches is underway. A network topology analysis takes inspiration by [2]. [2] and underlines how a given topological network asset may improve network resilience in response to an accidental failure but may also expose the network to high vulnerabilities in the presence of malicious attacks. A simulative analysis in which a federation of multiple domain-specific simulators, [3] 
or the development of specific tools for the simulation of interdependent infrastructures, [4] is proposed. A service oriented risk analysis in which the problem is simplified, considering that CIs support and fulfill services [5, 6, 7].

There are also many studies, which investigate the reciprocal effect of interdependencies among Power grids and Telco networks. Many of the above studies are especially based on Power grid simulation and the SCADA system is not explicitly modeled, or it is considered in a very simple way. In this paper, we discuss the performance and rerouting calculations of a specific service delivered by the SCADA system, and their impact on the QoS of the power supplied to customers. In particular, we refer to the Fault Isolation and System Reconfiguration (FISR) service that detects and isolates faults in the Power distribution grid and then reconfigures the grid to supply the isolated customers. In delivering F ISR service, SCADA system, Telco network and Power grid act as a whole heterogeneous network. Here, we investigate the usability of the same discrete event simulator, NS2, which fits very well to represent the SCADA system and Telco network, also to represent the power grid, limited to the implementation of FISR service. To build realistic models we refer to an actual case study, named Reference scenario, defined with the expertise of Israel Electric Corporation (IEC) in the framework of the MICIE project [8].

\section{Reference Scenario}

A Reference scenario is needed to limit the extension of the real world to be included in the analysis, and to provide a concrete context of operation, concentrated on CI interdependencies. Reference scenario identifies the following items: a) services; b) the set of interconnected networks supporting such services (in terms of topologies, essential systems: i.e. Telco emergency power supply, cooling systems); c) interconnections and types of interconnections among networks and systems; d) sequences of possible adverse events (failures, attacks, congestions) that could impair the quality of such services (in terms of continuity, readiness, performances, time response) and may include e) operator procedures to implement services under consideration.

Figure 1 shows the top level view of our Reference scenario. Natural or internal (malicious or random) adverse events (left boxes) hit (specific components of) interdependent Electrical, Communication and Information Infrastructures (boxes 1, 2 and 3 inside the central dashed box) and may propagate to customers of the Medium Voltage (MV) grid in terms of interruption of power supply. The interdependent SCADA system, Telco network and MV power grid, under consideration to represent FISR services, are respectively included in boxes 3, 2, 1. FISR, performed by SCADA system by means of SCADA operator is a particularly critical service because its degradation affects the quality of power supplied to customers.

In Power grids, failures may cause de-energisation for a large number of power customers and need to be located, isolated and repaired quickly and safely. In Reference Scenario, FISR service is remotely operated by the SCADA operator, that implements a specific procedure (based on grid monitoring, loss of power sensing, Circuit breakers operations) by means of Remote Terminal Units (RTUs). For confidentiality reasons the networks considered in the paper are just realistic abstractions of the actual ones of a geographical district of Israel, specifically customized for MICIE EU Project [8] by IEC experts. 


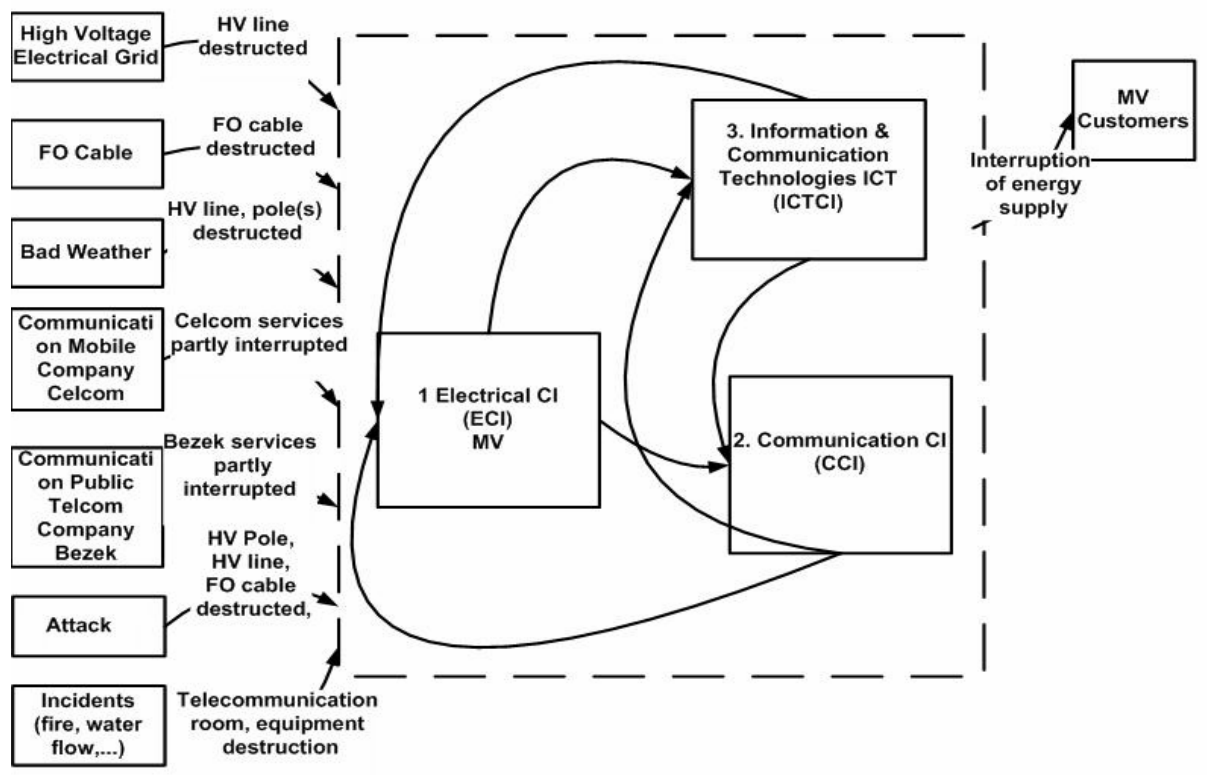

Fig. 1. Top level view of Reference scenario

\subsection{Power Grid}

Figure 2 shows the simplified view of the power distribution grid topology under consideration for FISR. It consists of a portion of a Medium Voltage (MV) grid at 22 $\mathrm{KV}$, energised by two substations, named TF and CB.

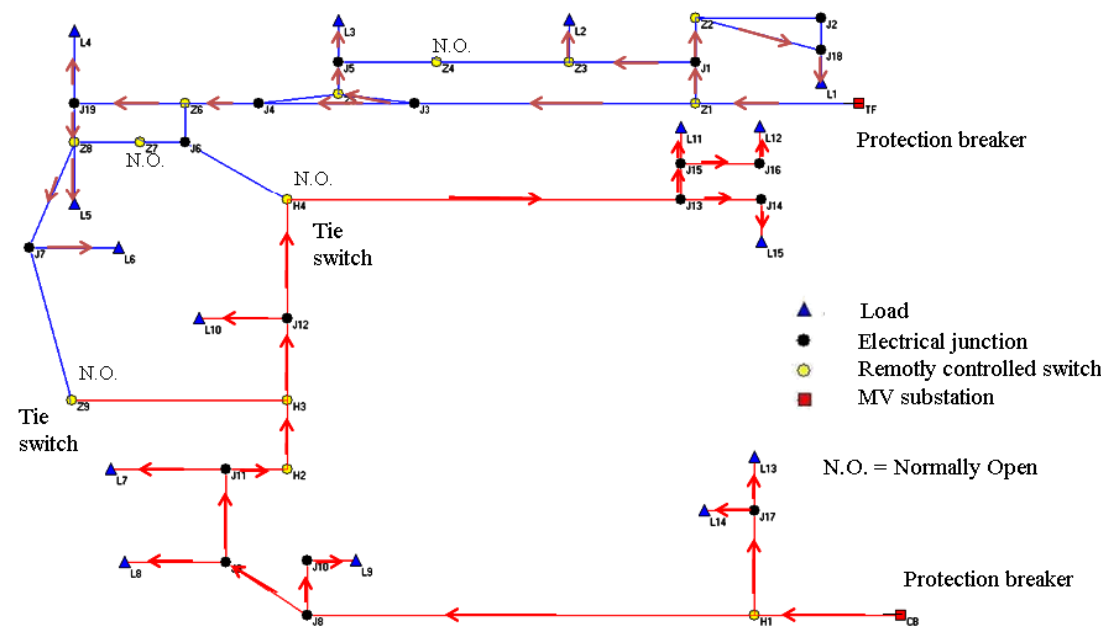

Fig. 2. A simplified view of MV power grid 
Each substation feeds different types of loads/customers (public, commercial, industrial), throughout electrical sections, connected one to another by Normally Close Circuit breakers. TF and CB substations include Protection breakers. In normal operative conditions, customers are energised by either TF substation or $\mathrm{CB}$ substation, by means of two sub grids, separated one from the other by two, Normally Open, Tie switches, remotely controlled by SCADA system. SCADA is also interconnected with the MV grid by means of its Remote Terminal Units to monitor the grid status and act on their Circuit breakers for connecting/isolating grid electrical sections.

\subsection{SCADA System}

Figure 3 shows the portion of SCADA system under consideration for FISR. From SCADA Control Centre (SCC), the operator remotely manages and controls in realtime the power grid of figure 2 , by means of RTUs.

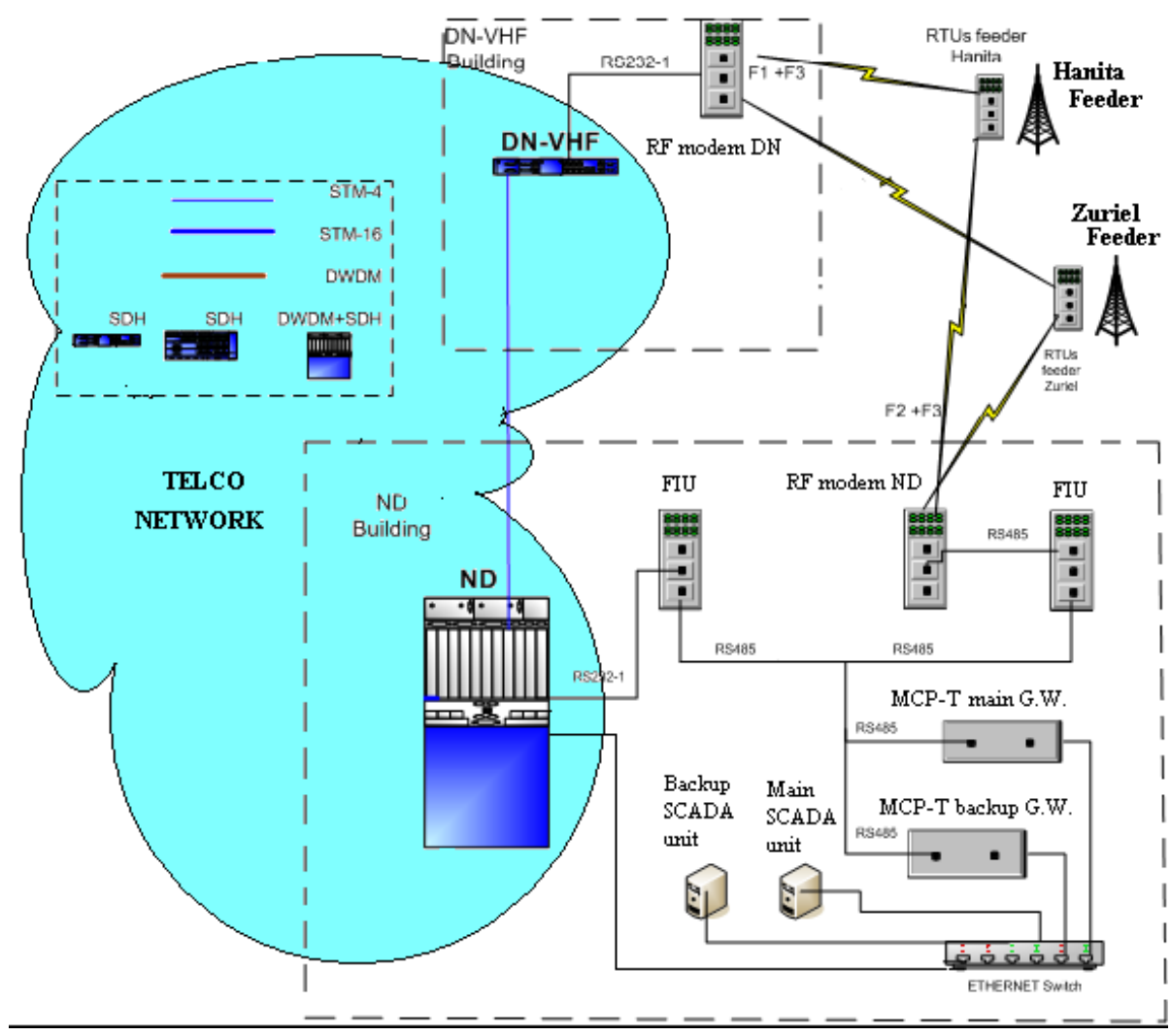

Fig. 3. SCADA system 
Particularly, the following devices belong to the SCADA system:

- MCP-T gateway which converts a proprietary Data Link Communication (DLC) protocol to the TCP/IP protocol. DLC protocol is designed for radio channels and allows multiple logical communication channels per communication medium. For DLC and TCP/IP protocols, every transmission is automatically accompanied by an ACK message, ensuring the integrity of the transmission.

- Field Interface Unit (FIU), dedicated to RTU interrogation and routing of data messages to/from SCC. FIU comprises a Radio Frequency (RF) Modem Interface (RF Modem ND), that includes two VHF radio units (F2, F3), that connect RTUs to SCC throughout either F2 or F3 channels.

- Store \& Forward (S\&F) Repeater DN which communicates upwards with the SCC (via the RF Modem and FIU) and downwards with the RTUs using the two RF channels (F1and F3).

- $\quad$ RTUs; there are 13 RTUs sites, of which 9 belong to TF and 4 to CB.

SCADA system is fully redundant. In case of failure of the main SCADA unit, the backup SCADA unit is enabled. The main communication path between SCC and the RTUs traverses the main Gateway and the main FIU. In case of failure on the main path, data are rerouted on the backup path that traverses the backup Gateway, the backup FIU, the Telco network (Point of Presence ND and Local eXchange DNVHF), $\mathrm{S} \& \mathrm{R}$ repeater and then reaches the RTUs. In case the primary RF channel is not available for any reason, the system switches to the alternative RF channel.

\subsection{Telco Network}

It is composed by three hierarchical layers:

- A Backbone layer, where Point of Presence (PoP) devices (figure 4) are connected one to another in a meshed topology. Its application is transport, so its primary concern is capacity. PoP is a multiservice optical platform that integrates several technologies including Synchronous Digital Hierarchy, Synchronous Optical Network (SDH/SONET) and Dense Wavelength Division Multiplexing (DWDM) in a single platform.

- A Local eXchange layer (LeX) (figure 4), the closest one to customers at the edge of the Transit eXchange layer, represents the point of access at lower bandwidth of Telco network. In this layer, IP traffic, with its inherently asymmetric and unpredictable nature, is predominant, especially with realtime applications.

- Between these two layers, lies the Transit eXchange layer (TeX) (figure 4) that grants scalable traffic in multi-ring topology. A TeX device is based on $\mathrm{SDH} / \mathrm{SONET}$ technology that aggregates data flows at different bit rates and retransmits them over long distances. It relies on optical rings constituted by ADM (Add Drop Multiplexer) and optical cables. ADMs perform signal multiplication (they gather many tributary signals and multiplex them into one signal at a higher rate), transmission over optical fibers and protection (by rerouting over the SDH ring in case of a single failure). 


\subsection{Heterogeneous Network Supporting FISR Service}

SCADA system, MV power grid and Telco network constitute a single heterogeneous network that supports FISR service, as shown in figure 4. In figure 4, a box bounds the main SCADA devices (except RTUs and radio links), a box bounds Telco devices that support a SCADA redundant link, and a box bounds the Power grid. There are several interconnections among these networks. SCADA system interacts with MV Power grid by means of SCADA RTUs (in figure 2 and inside the Power grid box of figure 4). Also SCADA devices, including RTUs and devices in Control Centers, are powered by the same MV Power grid. SCADA system also interacts with Telco network by means of the redundant link, which traverse PoP ND and LeX DN-VHF devices, and by the communication link between SCADA Control Centre and PoP $\mathrm{ND}$, as shown in figure 3 .

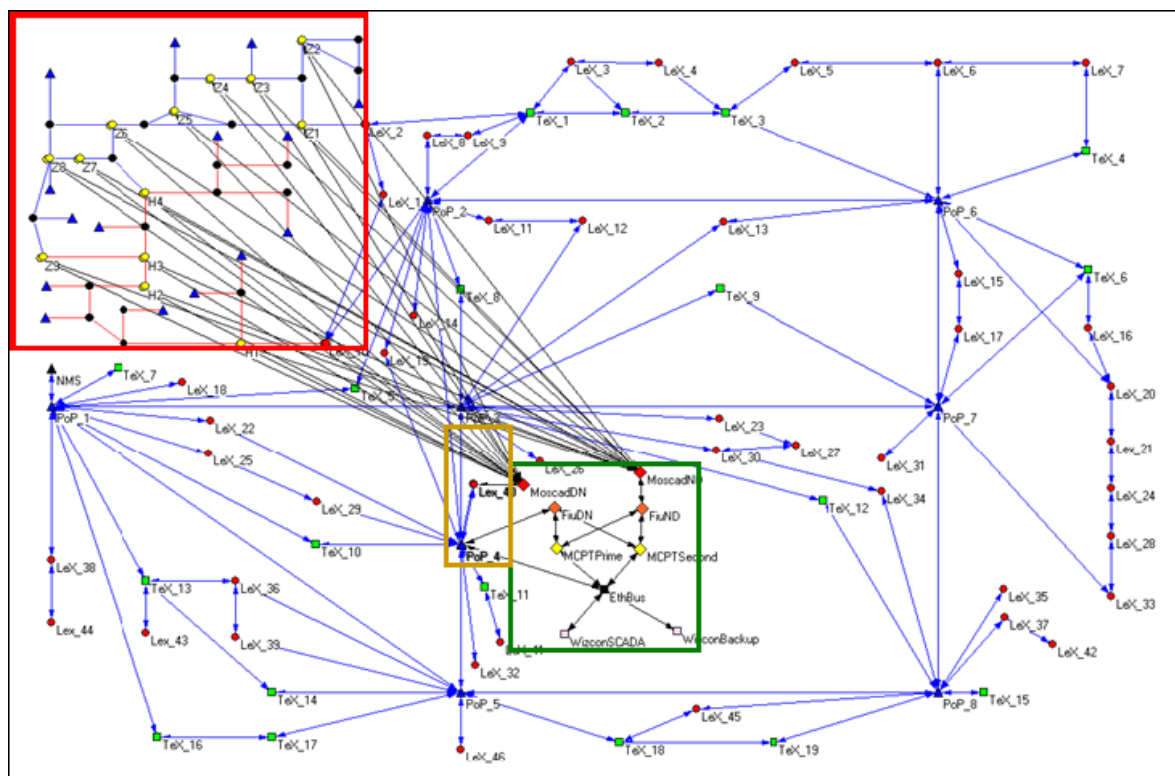

Fig. 4. Heterogeneous network supporting FISR service

\section{Discrete Event Simulation of Heterogeneous Networks}

QoS of FISR is computed by representing Power grid, SCADA system and Telco network as a single heterogeneous network in which continuous and discrete parameters coexist. Power grid mainly consists of elements that are typically modeled by continuous equations. Physical laws that dictate the behavior of electro mechanic elements of power grid are described by differential equations with some discrete dynamics needed to represent Circuit breakers. Then, to represent Power grids typically continuous simulators are used. The other way around, where SCADA and 
Telco networks are packet switching networks, then they need to be represented by discrete event simulators.

In our case, to compute QoS of F ISR, we need a full scale representation of SCADA and Telco network which act to implement FISR on the power grid and a proper representation of the power grid, as it is observable by the SCADA control centre (SCC), for the service under consideration. In general, the Power grid is observable by SCC in terms of bus voltages, line, generator \& transformer flows (MW, MVAR \& Amperes, transformer taps \& breaker status as well as other generator parameters (e.g. limits), frequency, requiring a full scale simulator for its representation. The concept of observability of power grid from SCC, limited to FISR service, can be simplified, by just representing the topology of the grid (substations, trunks, loads, junctions, RTU breakers), and the events involved in FISR service (remote On/Off operation of RTU breakers from SCC, presence/absence of the electrical flow from the feeding Substations to loads, according to electrical Junctions and RTU breaker positions, and occurrence of possible failures in any electrical section of the grid). For such a limited representation of the Power grid, we may resort to a discrete event simulator. Among discrete event simulators we choose NS2, one of the most widely used open source network simulators [9]. NS2 allows to simulate packet based local/wide area networks and wired/wireless networks and then it may well represent SCADA and Telco networks. First, we built a separate NS2 script for SCADA system, Telco network and power grid, than we integrate them to have a whole FISR model that relies on the heterogeneous network shown in figure 4.

\section{Quality of FISR Service}

The quality of FISR service is critical because it is strictly correlated to the quality of power supplied to customers. There are different indicators of the quality of power supplied to customers, such as the duration of power interruptions for a customer per year, the number of long/short power interruptions for customer per year, etc. Values of such indicators are typically regulated by a National Electric Authority. A timely actuation of FISR service, consequential to a permanent failure of the grid, reduces the outage duration and then contributes to keep indicators of quality of power supplied to customers within prefixed values. On the contrary a delayed actuation of FISR service gets the worst such indicators.

Here, we investigated FISR response time and correlated it with indicators for quality of power supplied to customers. FISR response time is considered a composed indicator because its values depend upon the values of indicators of performances and reliability of the networks that support such a service, here referred to as basic indicators. We investigated $s$-t dynamical path and $s$ - $t$ Round Trip Time as basic indicators of FISR. FISR response time is intended as the time between the occurrence of loss of power supply to customers (due to a grid failure) and the restoration of power supply to customers. We correlate it with the duration of grid outage and the percentage of customers affected by the outage. $S$ - $t$ dynamical path is intended as the path of nodes traversed by a packet from a source to a destination. It dynamically changes in consequence of network re-configuration caused by network congestion or link/node failures. It is computed between SCADA Control Centre and RTUs. S-t packet Round Trip Time (RTT) is intended as the packet transmission time, 
from a source to a destination plus the ACK time (from destination to source) - for TCP-IP protocols. It is computed between SCADA Control Centre and RTUs.

\section{Model of FISR Service}

FISR service is delivered by SCADA operators, according to procedures which may vary from one case to another. To represent FISR we account the following procedure [10]. Initially, MV power grid (Figure 2) is in its operative conditions. Then, randomly, a permanent failure occurs on any electrical section of the sub grid fed by either $\mathrm{CB}$ or $\mathrm{HF}$ substations. As a consequence the Protection breaker at the substation will trip. After two automatic reclosing attempts, the Protection breaker remains open, de-energizing all of the sub grid. The loss of power is sensed by each RTUs and using its backup battery, it opens the corresponding Circuit breaker,at this point, the "failure detection process" starts by re-closing progressively all breakers, starting from the one closest to the electrical substation up to the detected failed section.

On the attempt of the re-closure of the breaker at the head of the failed section, its RTU senses the loss of power and immediately re-opens the breaker and sends an alerting message to SCC, which acknowledges it. The re-opening of this breaker ensures that the failure is isolated. During such failure detection and isolation process all the customers included in the portion of the sub grid between the head of the failed section and the Normally Open Tie switches remain de-energised. At this point the "power restoration process" starts, remotely performed by the SCADA operator. The actual implementation of such a process depends upon the location of the failure inside the sub grid, and may include, if necessary, the closure of Normally Open Tie switches (thanks to request/response messages between SCC and RTUs) in order that all customers, except those included in the failed section, can be energised by the other substation. After the repair of the failed section, the grid can be reported to its initial configuration.

\subsection{Sub Model of Telco Network}

Telco network under consideration (figure 4) is composed of 74 nodes: 8 PoPs of the optical Backbone, 19 TeX, 46 LeX and 1 NMS (Network Management System).

Table 1 summarizes the main modeling assumptions. To generate a realistic traffic over the network, we consider that it hosts reliable traffic (i.e. for real time control devices and equipment, including SCADA) and less reliable traffic (i.e. enterprise traffic). We assume traffic generation according to two types of transport layer protocols of the IP (Internet Protocol) family, TCP (Transmission Control Protocol) and UDP (User Datagram Protocol) that provide network services for applications and application layer protocols. TCP and UDP perform their services by employing IP to route packets to their destination. TCP provides connection-oriented, reliable, bytestream packet delivery. UDP is a connectionless, unreliable message delivery protocol. The routing policy of the network nodes is a DV (Distance Vector) type. The queue of each link is Drop-Tail, which implements FCFS (First Come First Served) scheduling and drop-on-overflow buffer management. 
Table 1. Assumptions on Telco network

\begin{tabular}{llll}
\hline Link Type & Backbone (DWDM) & TeX (STM-16) & LeX (STM-4) \\
\hline $\begin{array}{l}\text { Capacity } \\
\begin{array}{l}\text { Source/Destination } \\
\text { Node }\end{array}\end{array}$ & PoP-PoP & $2.5 \mathrm{Gbps}$ & $600 \mathrm{Mbps}$ \\
$\begin{array}{l}\text { Traffic Type } \\
\text { Traffic Bit-Rate }\end{array}$ & PoP-TeX, TeX-TeX & PoP-LeX, TeX-LeX, \\
$\begin{array}{l}\text { Type of Agents } \\
\text { Number of Agents }\end{array}$ & CBR for UDP & TCP & LeX-LeX \\
100 for UDP & & TCP \\
\hline
\end{tabular}

\subsection{Sub Model of SCADA System}

The communication between main SCADA Centre and RTUs is implemented by a request/response application protocol that relies on the TCP/IP transport layer protocol. The radio links between RTUs on one side and RF modem ND or RF modem DN_S\&F on the other side, were implemented as ideal wireless links by means of a 'no loss' model (i.e. no shadowing, fading,...). Connections and IP traffic among Control Centre nodes and RTUs as well as among the nodes within the Control Centre were implemented with reference to [11]. Connections between the SCADA Control Centre and the RTUs were implemented by installing a TCP agent over each RTU, and a symmetrical TCP agent over each node representing the Control Centre. Then, on each TCP agent we locate a CBR (Constant Bit Rate) traffic source that transmits a packet of 255 bytes length, conforming to [11], with regular intervals of $30 \mathrm{sec}$ to simulate exchange of messages among RTUs and Control Centre nodes. The request -response mechanism between SCADA Centre and RTUs is implemented by means of the simulator's scheduler. Each Control Centre request to the RTU activates a CBR source on the RTUs as soon as the request is completely received. The TCP agent on the generic RTU will begin to transmit the response messages after a time interval, during which the RTU processes field data from the related electrical section of the grid. Table 2 summarizes the main assumptions.

Table 2. Assumptions on SCADA communication links

\begin{tabular}{lllll}
\hline Link Type & Ethernet & RS-485 & RS-232 & VHF-radio \\
& & & & \\
\hline Capacity & 100 Mbps & 19.2 Kbps & $19.2 \mathrm{Kbps}$ & $4.8 \mathrm{Kbps}$ \\
Source/ & SCADA - & MCP_T-FIU & RF modem - & RF modem - \\
$\begin{array}{l}\text { Destination } \\
\text { Node }\end{array}$ & MCP_T - PoP & FIU- RF modem & Telco Nodes & RTU \\
$\begin{array}{l}\text { Traffic type } \\
\text { Traffic bit-rate }\end{array}$ & DLC (TCP)+ TCP & DLC $(\mathrm{TCP})$ & DLC $(\mathrm{TCP})$ & DLC (TCP) \\
\hline
\end{tabular}

Occurrence of failures of electrical sections are detected and transmitted by RTUs and Substations to SCADA Control Centre. SCADA sub model represents the main path and the back up path between SCADA CC and RTUs. In case of failure of the main SCADA unit, the backup SCADA unit is enabled. In case of failure of the main FIU and/or of the main Gateway, data are rerouted on a backup path. Queue types and 
buffer sizes (the maximum number of packets can be stored before dropping) of links are defined as for Telco network sub model.

\subsection{Sub Model of Power Grid}

NS2 sub model of the grid consists of 49 elements: 2 substations, 13 Circuit breakers driven by correspondent SCADA RTUs, 19 junction nodes and 15 loads. In normal operation, the grid is separated into two sub-grids by means of Normally Open Tie switches. One sub grid, energized by TF substation, feeds 6 loads, while the other sub grid, energized by CB substation, feeds 9 loads. NS2 sub model of the MV power grid is interconnected with NS2 sub model of the SCADA system by means of Circuit breakers/RTUs. The status of Protection breakers at substations TF and $\mathrm{CB}$ is monitored by the SCADA Control Centre that can also actuate their remote reclosure.

To build a worthwhile NS2 sub model of the grid, careful attention has been paid to translate events and object of the electrical domain into adequate discrete modeling assumptions. The electrical current flowing from substations to loads is represented by CBR (Constant Bit Rate) packets transmitted from substation to loads by means of a static (source) routing protocol and a UDP transport layer protocol. UDP packets are sent almost continuously by an NS2 node representing the substation to NS2 nodes representing loads. On/off operations on the N.C. Circuit breakers, remotely driven by SCADA sub model, are represented by the occurrence of up/down events on the link that represents the electrical section posed immediately after (according to the direction of the electrical current in nominal conditions) the breaker. Along FISR implementation procedure, the direction of the electrical current on the grid may change upon operations on N. C. Circuit breakers or on N.O. Tie switches. Then the static routing policy of the NS2 model of the grid changes accordingly.

\section{Simulation Results}

In FISR model, we have grouped the occurrence of permanent failures on any electrical section of MV power grid as follows:

- failure in an initial section of the grid (bounded by the feeding substation and its closest RTU): the loads of failed sub-grid are energised by the other substation, up to the manual repair, that restores the initial configuration of the grid;

- failure in an intermediate section of the grid (bounded by two RTUs): the loads into this section are isolated, the loads bounded by failed the section and the tie switch are powered by the other substation, up to the manual repair, that restores the initial configuration of the grid;

- failure in a terminal section of the grid (bounded by RTU and loads): the loads of failed section are isolated, up to the manual repair, that restores the initial configuration of the grid. 


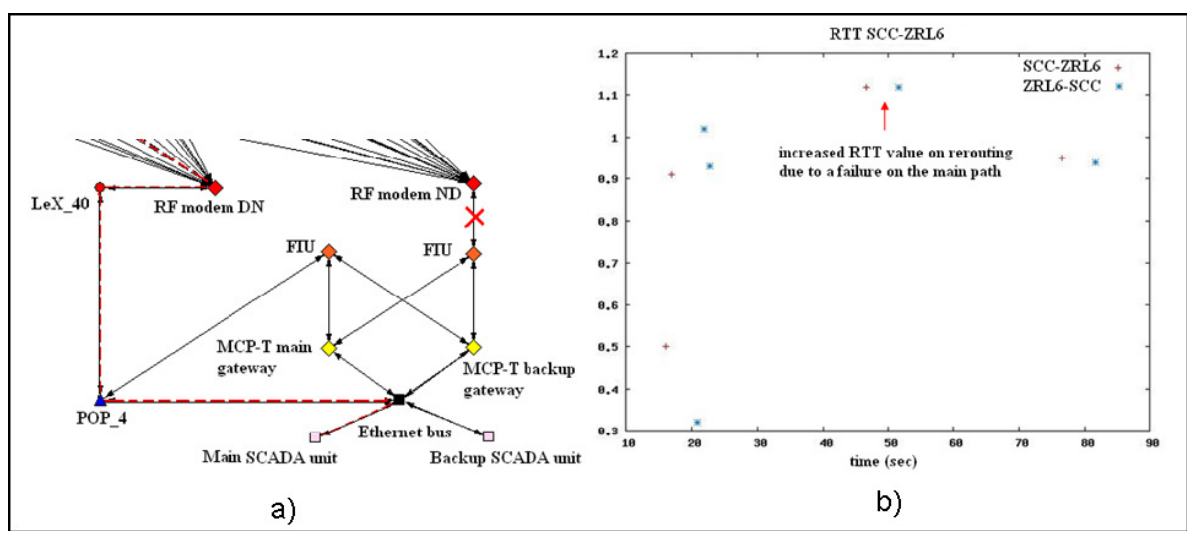

Fig. 5. a) S-t dynamical path and b) S-t RTT on failure in the main path of SCADA

Basic and composed indicators of QoS have been computed, under the following normal and failure conditions, by FISR model: case 1) normal condition of the SCADA system and Telco network; case 2) a failure of a SCADA element (i.e. the link between FIU and RF modem); case 3) a failure of a SCADA element (i.e. the link between FIU and RF modem) and a failure of a Telco network element (i.e. the link between PoP ND and LeX DN-VHF). Figure 5.a) shows, as dashed lines, the graphical representation of the $s$ - $t$ dynamical path between the Main SCADA unit of SCC and an RTU (ZRL6) in the presence of a failure of the link between FIU and RF modem ND of the SCADA system. Such a dynamical path is a back up path of the fully redundant SCADA system. Figure 5.b) shows, indicated by an arrow, the numerical value (sec.) of the $s$ - $t$ Round Trip Time of the messages between the Main SCADA unit of SCC and an RTU (ZRL6 RTU). This RTT value is greater than the corresponding RTT value with no failure on SCADA elements.

We have computed FISR response time and we have correlated it with the duration of grid outage and the percentage of the customers which remain isolated from the feeding substation (affected customers). The percentage is computed respect to the total number of the customers of the grid.

Table 3 summarizes the values of FISR response time and the percentage of affected customers for the three possible locations of the permanent failure on the power grid, and in different operative conditions (cases 1, 2 and 3) of SCADA system and Telco network. The first column of the table reports the failure location (within an initial, intermediate and terminal electrical section of the grid) that requires the

Table 3. FISR response time and $\%$ of customers affected by power outage

\begin{tabular}{llllll}
\hline $\begin{array}{l}\text { Section } \\
\text { of failure }\end{array}$ & $\begin{array}{l}\text { Response } \\
\text { time case } 1\end{array}$ & $\begin{array}{l}\text { Response } \\
\text { time case } 2\end{array}$ & $\begin{array}{l}\text { Response } \\
\text { time case } 3\end{array}$ & \multicolumn{2}{c}{ \% of affected customers } \\
\cline { 4 - 6 } & & & & Before FISR & After FISR \\
\cline { 5 - 6 } Initial & $18.4 \mathrm{sec}$. & $18.6 \mathrm{sec}$. & $>$ simulation time & 46.6 & 0 \\
Intermediate & $34.8 \mathrm{sec}$. & $35.2 \mathrm{sec}$. & $>$ simulation time & 26.6 & 0 \\
Terminal & $29.1 \mathrm{sec}$. & $29.4 \mathrm{sec}$. & $>$ simulation time & 26.6 & 6.6 \\
\hline
\end{tabular}


activation of FISR. Column 2 reports FISR time response with no failure of the SCADA system and Telco network (case 1). Columns 3 and 4 respectively report FISR time response under a failure of a SCADA element (case 2) and under a failure of a SCADA element and a Telco network element (case 3). FISR response time gets worst when passing from absence of failures up to a critical double failure. The percentage of the affected customers depends upon the section of the grid in which the failure is located. Failures in the initial section of the grid affect a higher percentage of customers. In case of a failure in the terminal section of the grid, there is a percentage of customers out of power service till the manual repair of the failure of the grid has been completed. The outage duration of the affected customers, in case 1 and 2, corresponds to the FISR response time plus the manual repair time when needed. Manual repair time is needed in case of failure in a terminal section of the grid. In case 3, FISR cannot be actuated remotely by SCC and the outage duration corresponds to the manual repair of the permanent failure of the grid.

\section{Conclusions}

The paper investigates the QoS delivered by interdependent infrastructures. Within a Reference scenario, the Quality of Fault Isolation and System Restoration (FISR) service, delivered by interconnected Power grid, SCADA system and Telco network has been investigated under nominal and failure conditions. We demonstrate the applicability, the advantages and the limits of using a unique discrete event simulator, to represent the three heterogeneous networks supporting FISR service. Basic and composed indicators of Quality of FISR have been computed and a correlation between them and Quality of Service to grid customers has also been discussed.

Acknowledgments. This research was partially motivated by EU projects MICIE http://www.micie.eu, IRRIIS http://www.irriis.org/ and the Italian MIUR project CRESCO http://www.cresco.enea.it/

\section{References}

1. Rinaldi, S.M., Peerenboom, J.P., Kelly, T.K.: Identifying, Understanding, and Analyzing Critical Infrastructure Interdependencies. IEEE Control Systems Magazine 21(6) (2001)

2. Albert, R., Barabasi, A.L.: Statistical Mechanics of Complex Networks. Review of Modern Physics 74, 47-97 (2002)

3. Hopkinson, K., Wang, X., Giovanini, R., Thorp, J., Birman, K., Coury, D.: EPOCHS: A Platform for Agent-Based Electric Power and Communication Simulation Built From Commercial Off-the-Shelf Components. IEEE Trans. on Power Systems 21(2) (2006)

4. Dudenhoeffer, D., Permann, R., Manic, M.: CIMS: A framework for infrastructure interdependency modeling and analysis. In: Proc. of Winter Simulation Conference (2006)

5. Bonanni, G., Ciancamerla, E., Minichino, M., Clemente, R., Iacomini, A., Scarlatti, A., Zendri, E., Terruggia, R.: Exploiting stochastic indicators of interdependent infrastructures: the service availability of interconnected networks. In: Martorell, S., Soares, C.G., Barnett, J. (eds.) Safety, Reliability and Risk Analysis: Theory, Methods and Applications, vol. 3. Taylor \& Francis, London (2009) 
6. Capodieci, P., et al.: From heterogeneous modeling and analysis to an on line prediction tool to improve QoS of interdependent networks. Electricity, Tel Aviv, Israel (2009)

7. Bobbio, A., Ciancamerla, E., Diblasi, S., Iacomini, A., Mari, F., Melatti, I., Minichino, M., Scarlatti, A., Terruggia, R., Tronci, E., Zendri, E.: Risk analysis via heterogeneous models of SCADA interconnecting Power Grids and Telco Networks. In: CRiSIS 2009, Toulouse, France, October 19-22 (2009)

8. http://www.micie.eu

9. http://www.isi.edu/nsnam/ns/

10. http://www.itsinfo.com/doc/moscad_electric_power.pdf

11. IEC870-5-1 01/105 guidelines 